\title{
EL ERROR DE DIAGNÓSTICO DE LA DERECHA CHILENA Y SU ENCRUCIJADA ACTUAL
}

\author{
Cristóbal Rovira Kaltwasser \\ Universidad Diego Portales, Chile \\ Centro de Estudios de Conflicto y Cohesión Social (COES), Chile
}

\begin{abstract}
Resumen: El estallido social de fines del año 2019 ha abierto una serie de conflictos al interior de la derecha chilena. Ahora bien, estos conflictos no surgieron de la noche a la mañana, sino que tienen sus orígenes en la existencia de distintas interpretaciones respecto de cómo es la sociedad chilena de hoy en día y de cuáles son las mejores estrategias para desarrollar un perfil electoral competitivo. Para comprender esta problemática, en este artículo se ofrece una discusión teórica sobre la tensión entre moderación y radicalización que experimenta la derecha, para luego proceder a revisar evidencia empírica del caso chileno sobre las transformaciones de las preferencias de la sociedad y las estrategias electorales que ha venido desarrollando la derecha. Interesa argumentar que el segundo gobierno de Sebastián Piñera, que ganó la elección presidencial el año 2017, se
\end{abstract}

Cristóbal Rovira es profesor titular de la Escuela de Ciencia Política de la Universidad Diego Portales, Santiago, Chile. Es investigador asociado del Centro de Estudios de Conflicto y Cohesión Social (COES), Santiago, Chile. Dirección: Universidad Diego Portales, Manuel Rodríguez 450, Santiago, Chile, CP 8320000. Email: cristobal.rovira@mail.udp.cl.

Este artículo retoma ideas elaboradas en publicaciones previas sobre la derecha chilena, en particular Rovira Kaltwasser $(2017,2019)$, y Madariaga y Rovira Kaltwasser (2019). Quisiera agradecer a Ariel Becerra y Lisa Zanotti por su ayuda en la sistematización de los datos que se presentan en este artículo. A su vez, agradezco los comentarios tanto de Aldo Mascareño como de Raimundo Frei, y también el apoyo del Fondo Nacional de Desarrollo Científico y Tecnológico de Chile (proyecto Fondecyt 1180020) y del Centro de Estudios de Conflicto y Cohesión Social (COES, ANID/ Fondap/15130009). 
basó en un profundo error de diagnóstico sobre el país. Esto no solo facilitó la irrupción del estallido social, sino que también ha pavimentado el camino para que se agraven las tensiones al interior de la derecha. De ser cierta la tesis que acá se plantea, lo más probable es que en un futuro cercano deje de existir una sola coalición de derecha, posibilitándose así la conformación de pactos gubernamentales inéditos hasta ahora.

Palabras Clave: Chile, derecha, estallido social, moderación, populismo, radicalización

RECIBIDO: enero 2020 / ACEPTADO: marzo 2020

\section{THE DIAGNOSIS ERROR OF THE CHILEAN RIGHT-WING AND THE CROSSROADS IT FACES TODAY}

AвSTRACT: The social unrest that occurred at the end of 2019 has caused a series of conflicts within the Chilean right-wing. These conflicts did not appear overnight, rather, they originate from a series of different outlooks on today's Chilean society as well as on the best strategies to develop a competitive electoral profile. In order to understand this issue, we offer herein a theoretical discussion on the tension between moderation and radicalization within the right-wing, and thereafter an analysis of the empirical evidence of the Chilean case regarding the transformation of the society's preferences as well as the electoral strategies the right-wing has developed. Interestingly, the second-term government of Sebastián Piñera, who won the election in 2017, was based on a sheer error in the diagnosis of the country's situation. This not only facilitated the social unrest, but also paved the way to increased tension within the right-wing. Should the thesis proposed herein be correct, it is likely that in the near future a single right-wing coalition will cease to exist, therefore opening the way to unprecedented political alliances.

KeYwords: Chile, right-wing, social unrest, moderation, populism, radicalization

ReCEIVED: January 2020 / ACCEPTED: March 2020

\section{INTRODUCCIÓN}

D esde la recuperación de la democracia chilena en 1989 hacia adelante, los partidos políticos de derecha se han mantenido unidos al formar parte de una misma coalición electoral, la cual ha logrado conquistar el Poder Ejecutivo bajo el liderazgo de Sebastián Piñera en dos ocasiones: los años 2009 y 2017. Sin embargo, en el presente ar- 
tículo me interesa mostrar que el más reciente triunfo presidencial de la derecha chilena fue de la mano con un profundo error de diagnóstico sobre la situación del país. En términos simples, este error de diagnóstico descansa en dos argumentos que se entrelazan. En primer lugar, la incapacidad de gran parte de la derecha de darse cuenta de que desde hace un tiempo que la mayoría de la ciudadanía demanda tanto posturas más liberales en temas morales, como también la construcción de un modelo socialdemócrata que garantice un piso mínimo de seguridad para el conjunto de la población. En segundo lugar, la creencia de parte de un sector de la derecha de que para mantener un perfil electoralmente competitivo no es necesario moderar las posturas programáticas hacia el centro político, sino que más bien hay que radicalizarlas hacia la derecha para así conectar con las ideas e intereses de un sector supuestamente amplio de la sociedad chilena que no se siente representado por el sistema político.

Este error de diagnóstico ciertamente terminó por facilitar el así llamado 'estallido social' de fines del año 2019, el cual tomó absolutamente por sorpresa al gobierno en particular y a la clase política en general. Como se verá más adelante en detalle, el error de diagnóstico de la derecha chilena obedece no solo a la ceguera de una parte importante de sus dirigentes respecto de las demandas de la ciudadanía, sino también a disputas internas que llevaron a que el gobierno de Sebastián Piñera, electo en 2017, opte por detener - e incluso revertir - un proceso de moderación programática que la derecha chilena venía experimentando desde fines de la década de 1990 en adelante, el cual había implicado un acercamiento a posturas de centro. Esta decisión terminó siendo fatídica para la derecha por dos motivos: por un lado, profundizó su brecha en relación al parecer de la sociedad y, por otro lado, agravó la tensión interna entre quienes pujan por una moderación hacia el centro y quienes presionan por una radicalización hacia posturas de derecha extrema.

Ahora bien, la tensión entre moderación y radicalización no es algo nuevo al interior de la derecha chilena ni algo que solo le suceda a esta última. Existe una amplia literatura en política comparada que demuestra que en Europa occidental los partidos políticos de derecha convencional se ven crecientemente desafiados por la irrupción de fuerzas populistas de derecha radical y, por lo tanto, es importante tomar en consideración esta literatura para comprender de mejor forma la encrucijada en la cual 
se encuentra la derecha chilena. De hecho, la situación hoy en día de la derecha del país y los desafíos que le tocará enfrentar en un futuro próximo dan pie para pensar que la mantención de la actual coalición electoral es altamente improbable. En otras palabras, todo indica que más temprano que tarde la derecha chilena se fragmentará en distintas fuerzas políticas y esto puede dar paso para la formación de coaliciones electorales que no han acontecido desde el retorno de la democracia.

El resto de este artículo se divide en cinco apartados. En primer lugar, se ofrece una breve revisión de la literatura académica sobre la transformación de la derecha en Europa occidental, la cual enfatiza la existencia de una creciente tensión entre moderación y radicalización, que ha traído consigo tanto la fragmentación de la derecha como el aumento de las pugnas al interior del bloque de derecha. Después de esto, a través de datos de opinión pública del electorado chileno, se muestra su gradual movimiento hacia posturas cada vez más liberales en temas morales y cada vez más alejadas del modelo de libre mercado. A continuación, se ofrece un análisis empírico de los programas de gobierno que ha elaborado la derecha chilena para las campañas presidenciales. Aquí se demuestra que el proceso de moderación programática que venía experimentando la derecha chilena se vio interrumpido en la campaña del año 2017, cuando Sebastián Piñera toma en consideración las posturas defendidas por quienes intentaban introducir una agenda de derecha populista radical. La cuarta sección se centra en la situación actual de la derecha chilena, y plantea que su error de diagnóstico facilitó la irrupción del estallido social y está provocando dinámicas centrífugas que probablemente conllevarán al ocaso de la coalición electoral en un futuro cercano. Finalmente, el artículo concluye ofreciendo un breve resumen del argumento central y sus implicaciones para el futuro tanto de la derecha chilena como del sistema político del país.

\section{EL DILEMA DE LA DERECHA EN EUROPA OCCIDENTAL: MODERACIÓN VERSUS RADICALIZACIÓN}

No hay duda alguna de que los sistemas políticos de Europa occidental han sufrido importantes transformaciones en las últimas décadas, siendo quizás la más revelante la irrupción de dos nuevas fuerzas políticas que se han consolidado en la gran mayoría de los países de la región: 
por un lado, los partidos verdes con una agenda a favor del medio ambiente y el multiculturalismo y, por otro lado, los partidos populistas de derecha radical centrados en la antiinmigración y la promoción de un nacionalismo chauvinista (Mudde 2007). Lo interesante de este nuevo conflicto que se ha ido cristalizando a lo largo y ancho de Europa occidental es que su origen descansa, antes que nada, en una disputa posmaterialista.

Para comprender esto, es preciso subrayar que el cientista político Ronald Inglehart $(1977,1990)$ se hizo famoso por postular y demostrar que el sostenido crecimiento económico experimentado por las sociedades europeas trajo consigo una 'revolución silenciosa': una gradual transformación de la estructura de valores, de modo tal que las demandas materiales fueron perdiendo peso y lentamente comenzaron a cobrar mayor fuerza las demandas de tipo posmaterial, tales como la igualdad de género, el respeto al medio ambiente y la diversidad cultural. Los estudios de Inglehart comprueban que las nuevas generaciones son las más proclives a expresar este cambio valórico, lo cual viene a representar un desafío mayúsculo para los partidos políticos convencionales que estaban acostumbrados a competir fundamentalmente en el clásico eje derecha-izquierda en términos socioeconómicos y no habían puesto mucho énfasis en disputas en el eje derecha-izquierda en términos socioculturales.

En consecuencia, la materialización de la 'revolución silenciosa' de Inglehart puso en marcha una dinámica de cambio en los sistemas de partidos de Europa occidental. Mientras que los partidos socialdemócratas comenzaron a adoptar posturas más liberales en temas morales y a dejar de lado posturas más estatistas en temas económicos, los partidos de derecha convencional — tales como los partidos conservadores y cristianodemócratas - también se vieron forzados a repensar sus agendas programáticas para adaptarse a un electorado que lenta pero consistentemente presionaba por dejar de lado posturas moralmente conservadoras y marcadamente nacionalistas (Kitschelt 1994; Kriesi et al. 2008; Gidron y Ziblatt 2019). Para la derecha convencional, el corolario de esta situación ha sido una gradual moderación ideológica, la cual se puede observar con marcada nitidez en dos liderazgos recientes: Angela Merkel del Partido Demócrata Cristiano en Alemania y David Cameron del Partido Conservador en Reino Unido. Ambas figuras bus- 
caron defender posturas a favor tanto del multiculturalismo como de la temática ecológica y abrieron el debate sobre el matrimonio igualitario en sus respectivos partidos, de modo tal que una parte importante de sus parlamentarios terminaron aprobando iniciativas en tal sentido en los congresos de Alemania y Reino Unido, respectivamente.

Si bien es cierto que este proceso de moderación programática de los partidos convencionales de la derecha en Europa occidental ha sido más rápido y profundo en algunos países que en otros, en prácticamente todos se puede observar una tendencia hacia la adopción de posiciones más liberales en temas socioculturales. No obstante, este proceso de moderación programática fue debilitando los vínculos existentes con ciertos segmentos del electorado con posturas más extremas, de modo que se fue generando un nicho de votantes que más temprano que tarde ha terminado siendo cautivado por los partidos populistas de derecha radical. Tal como indica Piero Ignazi (1992) en un célebre texto, los partidos populistas de derecha radical deben ser pensados como el resultado de una 'contrarrevolución silenciosa'. Se trata de una familia de partidos políticos que surgen gracias a la promoción de ideas que existen en un segmento de la ciudadanía que no se siente representado por el giro posmaterial de los partidos de derecha convencional.

Dado que los conceptos de 'populismo' y de 'populismo de derecha radical' son bastante debatidos, antes de continuar resulta conveniente realizar una breve reflexión sobre su uso en este artículo. En línea con varios trabajos académicos (Mudde 2007, 2013; Mudde y Rovira Kaltwasser 2013, 2017), acá se plantea que los partidos populistas de derecha radical se caracterizan por compartir tres atributos ideológicos: autoritarismo, nativismo y populismo. En primer lugar, la noción de autoritarismo no hace referencia al ataque directo a la democracia, sino más bien a la defensa de una sociedad estrictamente ordenada y a la demanda de 'mano dura' frente a todo aquello que se considera como conductas desviadas. En segundo lugar, la noción de nativismo descansa en la idea de que en el Estado deben habitar exclusivamente miembros del grupo nativo ('la nación') y de que los elementos no nativos ('extranjeros') representan una amenaza fundamental para la homogeneidad de la comunidad política. En tercer y último lugar, el populismo debe ser entendido como una ideología política que plantea no solo que la sociedad está dividida entre 'el pueblo puro' y 'la elite 
corrupta', sino también la idea de que la política debe ser expresión de la voluntad general.

La conjugación de autoritarismo, nativismo y populismo le otorga un rasgo distintivo a esta familia de partidos políticos por sobre la derecha convencional, generando así una modificación en la lógica de la competencia electoral. En efecto, una vez que emergen y se consolidan los partidos populistas de derecha radical, los partidos de derecha convencional comienzan a experimentar un dilema que no tiene fácil solución: por un lado, pueden mantener la senda de la moderación programática, que inevitablemente conlleva una pérdida de los electores más extremos que optan por votar a los partidos populistas de derecha radical o, por otro lado, pueden decidir girar hacia posturas programáticas más extremas, lo cual inevitablemente traerá consigo una pérdida de electores más moderados que prefieren votar por partidos que ofrecen una agenda más cercana al centro político (Bale 2003). La situación actual en Europa occidental está fuertemente marcada por esta encrucijada que sufren los partidos de la derecha convencional, los cuales han ido perdiendo su capacidad para representar a amplios sectores de la sociedad y observan con alarma cómo se fragmenta el espacio electoral de derecha en distintos grupos ideológicos que son difícilmente reconciliables.

Este breve resumen de la transformación programática de la derecha convencional en Europa occidental nos ayuda a comprender que la situación actual de la derecha chilena no es necesariamente singular. Por el contrario, como veremos más adelante, la encrucijada en que se encuentra la derecha en Chile tiene importantes similitudes con lo que sucede en varios países de Europa occidental. Sin embargo, también es cierto que el sistema político chileno tiene algunas peculiaridades que se deben tomar en consideración. Es por ello que en la próxima sección se hace una revisión de datos de opinión pública para reflexionar en torno a los cambios que ha venido experimentando el electorado chileno desde la transición a la democracia en 1989 hacia adelante.

\section{LA GRADUAL TRANSFORMACIÓN DEL ELECTORADO CHILENO}

Demostrar la evolución de las preferencias del electorado chileno en el tiempo es un ejercicio bastante engorroso, ya que existen muy pocas preguntas de opinión pública que se mantienen de manera sistemática 
por largos períodos. Sin embargo, al hacer una sistematización de los datos existentes es posible obtener cierta información que revela que, desde el retorno de la democracia, el electorado chileno ha venido experimentando importantes cambios que van en línea con los argumentos de la 'revolución silenciosa' planteada por Ronald Inglehart. De hecho, existe amplio consenso respecto de que el país ha vivido un proceso de expansión económica desde 1990 en adelante y, por lo tanto, no es descabellado pensar que la sociedad ha vivido relevantes transformaciones culturales no solo producto de este proceso de modernización económico, sino también - y sobre todo- debido a la emergencia de nuevos actores que han tenido la capacidad de politizar demandas de corte sociocultural (basta pensar en la presencia y el impacto que ha tenido el movimiento feminista en Chile últimamente).

En una publicación previa (Rovira Kaltwasser 2019) ya sistematicé datos de la Encuesta Mundial de Valores, los cuales reflejan que a inicios de la transición a la democracia la sociedad chilena era sumamente conservadora frente a temas morales como el aborto, el divorcio y la homosexualidad, pero esto ha ido cambiando gradualmente con el pasar de los años y hoy en día la sociedad chilena es mucho más liberal que antes. Una conclusión similar se ofrece en el reciente reporte sobre los diez años de la auditoría a la democracia en Chile, en donde se plantea que

la población chilena ha experimentado un acelerado cambio cultural. En un período de diez años la sociedad transitó hacia representaciones más igualitarias sobre los roles de género, aumentó la tolerancia hacia la homosexualidad y el aborto, y también la valoración de la inmigración. (PNUD 2020, 97)

Para reforzar los hallazgos de investigaciones previas, en este artículo me interesa agregar dos evidencias empíricas provenientes de las encuestas de la última década del Latin American Public Opinion Project (LAPOP). Por un lado, la pregunta sobre la justificación del aborto cuando peligra la salud de la madre ha venido creciendo en apoyo de manera sistemática: mientras en el año 2012 un poco más de la mitad de la población estaba de acuerdo con esto, hoy en día aproximadamente el $80 \%$ de la población piensa así (ver Gráfico 1). Por otro lado, en la última década también ha venido creciendo el número de personas que están a favor de que personas del mismo sexo puedan tener el derecho a 
casarse. Usando una escala en la que 1 implica una firme desaprobación de esta idea y 10 implica una firme aprobación de esta idea, el promedio ha variado de 4,5 en 2012 hacia 6,4 en la última medición efectuada en los años 2018 y 2019 (ver Gráfico 2).

Gráfico 1. ¿CREE USTED QUE SE JUSTIFICARÍA LA INTERRUPCIÓN DEL EMBARAZO, O SEA, UN ABORTO, CUANDO PELIGRA LA SALUD DE LA MADRE? (PORCENTAJE)

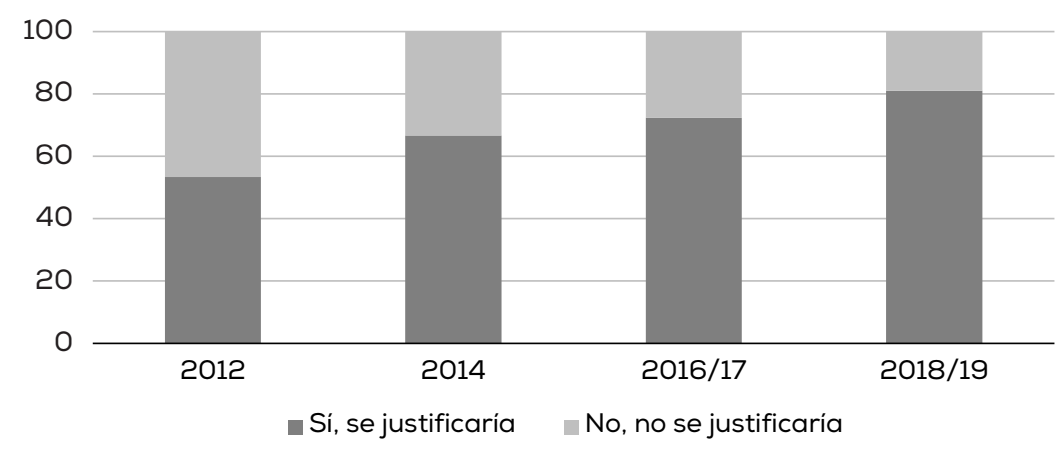

Fuente: elaboración propia en base a los datos del Latin American Public Opinion Project (LAPOP).

Gráfico 2. ¿CON QUÉ FIRMEZA APRUEBA O DESAPRUEBA QUE LAS PAREJAS DEL MISMO SEXO PUEDAN TENER EL DERECHO A CASARSE? PROMEDIO EN UNA ESCALA DONDE 1 SIGNIFICA 'DESAPRUEBA FIRMEMENTE' Y 10 SIGNIFICA 'APRUEBA FIRMEMENTE'

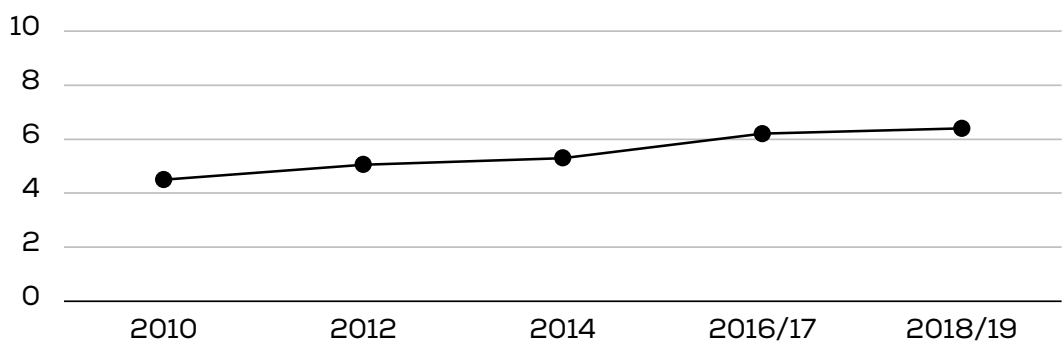

Fuente: elaboración propia en base a los datos del Latin American Public Opinion Project (LAPOP).

En consecuencia, los datos de opinión pública existentes para Chile permiten argumentar que el electorado del país ha seguido un derrotero similar al de Europa occidental, en el sentido de que a nivel societal 
gradualmente se han ido consolidando posturas más liberales en temas culturales. De manera simultánea, el electorado chileno ha experimentado una segunda transformación relacionada con temas socioeconómicos: paulatinamente ha venido aumentando la demanda por una mayor presencia del Estado en la economía y también ha crecido el malestar hacia los niveles de desigualdad existentes. Al igual como sucede con los temas socioculturales, es bastante difícil encontrar datos de opinión pública que permitan rastrear de modo sistemático las preferencias socioeconómicas del electorado desde la recuperación de la democracia en adelante. Sin embargo, una revisión de las encuestas existentes da cuenta de esta segunda transformación del electorado chileno hacia posturas que se alejan del ideario de la derecha y se acercan al pensamiento de la izquierda.

Gráfico 3. ¿CUÁN JUSTA CREE UD. QUE ES LA DISTRIBUCIÓN DEL INGRESO EN CHILE? (PORCENTAJE)

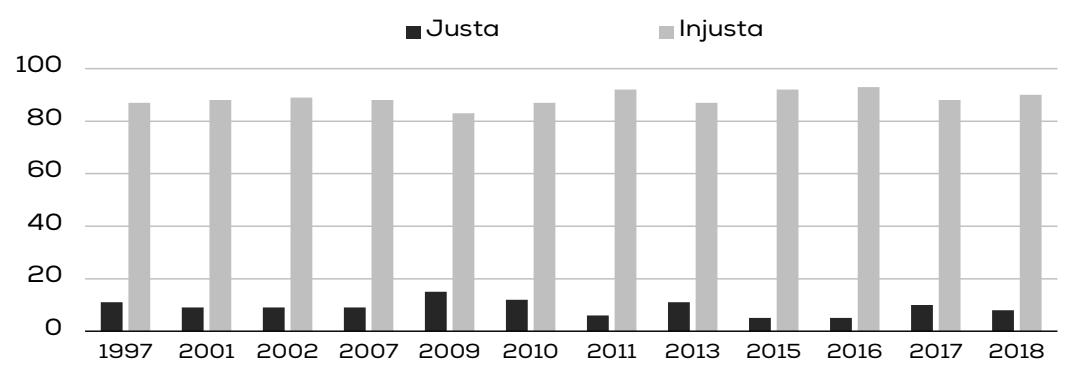

Fuente: elaboración propia en base a los datos de Latinobarómetro.

Un primer tema que es central obedece a la opinión de la sociedad frente al tema de la desigualdad. La evidencia empírica revela que Chile es un país que en perspectiva comparada presenta muy elevados niveles de desigualdad socioeconómica (PNUD 2017). A su vez, los datos de opinión pública sobre este tópico reflejan dos tendencias relevantes. Por un lado, prácticamente no existe variación en el tiempo con respecto a la creencia de que los recursos están repartidos de forma sumamente inequitativa. Tal como se puede observar en el Gráfico 3, de manera constante alrededor de un $85 \%$ de la ciudadanía opina que la distribución del ingreso es sumamente injusta en el país. Por otra parte, las últimas mediciones disponibles revelan que ha venido aumentando la cantidad de 
personas que mantienen que las privatizaciones de las empresas públicas no han sido beneficiosas para el país (ver Gráfico 4). De hecho, la serie histórica sobre este dato indica que tan solo existe una medición (año 1998) en donde la mayoría de la población opina que las privatizaciones de las empresas públicas sí han sido beneficiosas para el país.

Gráfico 4. LAS PRIVATIZACIONES DE LAS EMPRESAS PÚBLICAS HAN SIDO BENEFICIOSAS PARA EL PAÍS (PORCENTAJE)

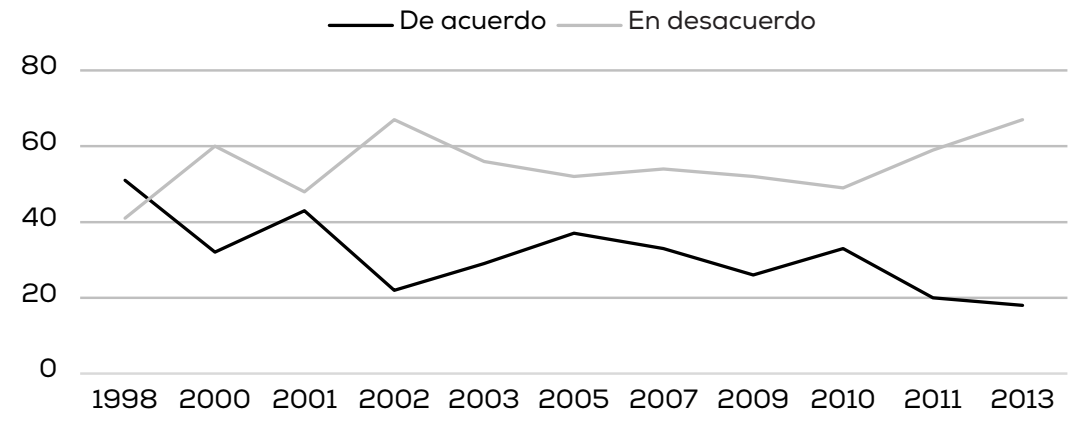

Fuente: elaboración propia en base a los datos de Latinobarómetro.

Ahora bien, la percepción de la existencia de una distribución del ingreso muy injusta y la evaluación negativa de las privatizaciones de las empresas públicas no quiere decir que la población quiera echar abajo el modelo económico imperante. En efecto, datos disponibles indican que, pese a ciertas variaciones en el tiempo, una gran mayoría de la sociedad sostiene que la economía de mercado es el único sistema con el que Chile puede llegar a ser desarrollado (ver Gráfico 5). La demanda central de la ciudadanía no consiste en la implementación del modelo ‘cubano' o ‘venezolano', sino más bien en la construcción de un modelo socialdemócrata que otorgue un piso mínimo de seguridad para el conjunto de la población y no solo para una minoría económicamente privilegiada. El problema de fondo es que cada vez existe menos tolerancia a los niveles de desigualdad existentes en el país. Tal como se puede observar en el Gráfico 6, mientras en el año 2000 aproximadamente la mitad de la población era de la opinión de que es injusto que aquellos que pueden pagar más tengan acceso a una mejor salud/educación para sus hijos, esta cifra ascendió a aproximadamente un $65 \%$ en el año 2016. 
Gráfico 5. LA ECONOMÍA DE MERCADO ES EL ÚNICO SISTEMA CON EL QUE CHILE PUEDE LLEGAR A SER DESARROLLADO (PORCENTAJE)

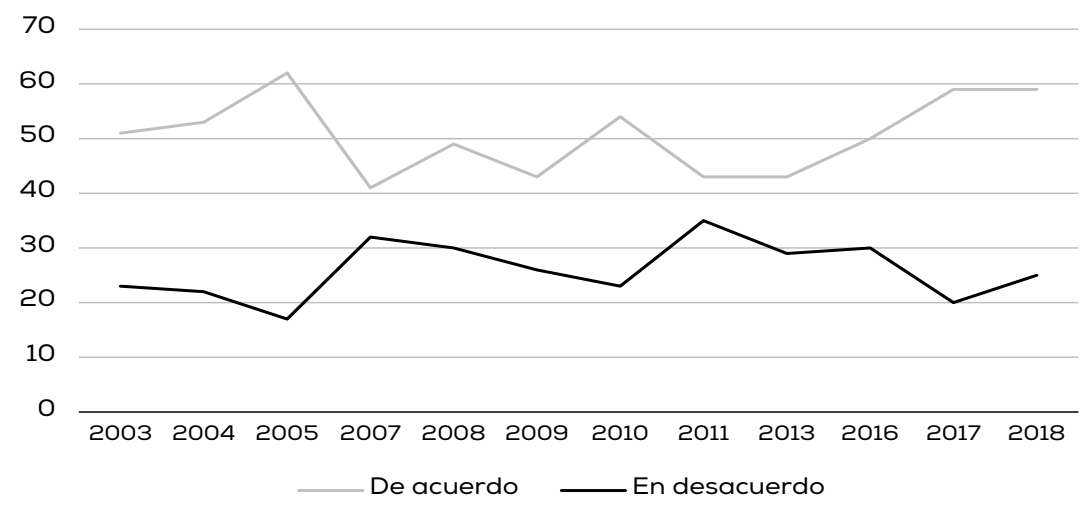

Fuente: elaboración propia en base a los datos de Latinobarómetro.

Gráfico 6. PORCENTAJE DE LA POBLACIÓN QUE ESTÁ EN DESACUERDO CON LA EXPRESIÓN 'ES JUSTO QUE AQUELLOS QUE PUEDEN PAGAR MÁS TENGAN ACCESO A UNA MEJOR SALUD/EDUCACIÓN PARA SUS HIJOS'

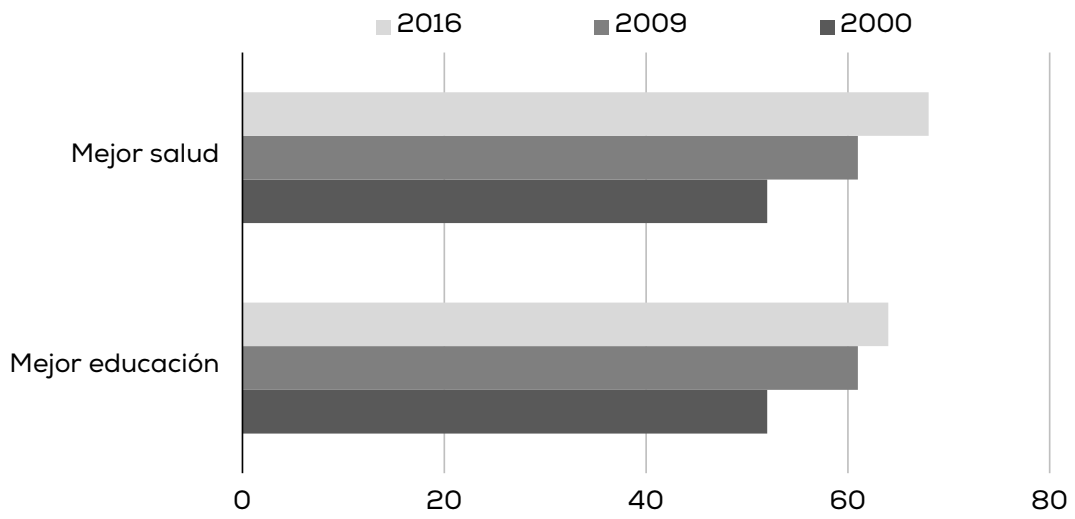

Fuente: elaboración propia en base a PNUD (2017, 323).

Sin duda que este cambio de la sociedad chilena hacia una demanda por mayor igualdad socioeconómica tiene diversas causas. No obstante, una parte importante de la literatura académica ha resaltado que un elemento central es la politización de la desigualdad por parte de nuevos actores sociales, los cuales han podido ir generando suficientes 
recursos organizacionales como para movilizar a la ciudadanía, realizar ciclos de protesta e incluso formar nuevos partidos políticos que levantan a sus propios candidatos y programas para las elecciones (Donoso y Von Bülow 2017; Roberts 2016). Son justamente estos nuevos actores sociales los que han tenido la capacidad de modificar el debate público al politizar la desigualdad y presionar por la implementación de políticas públicas redistributivas. Como bien indica Daniel Mansuy (2016), la creciente politización de la desigualdad social y la aparición de demandas por cambios profundos tomaron por sorpresa a gran parte de la derecha, ya que esta última no solo creyó que el modelo de desarrollo económico imperante contaba con una legitimidad masiva a nivel de la ciudadanía, sino que además nunca invirtió mucho tiempo ni energía en legitimarlo socialmente, puesto que los amarres institucionales de la Constitución fácticamente imposibilitan la realización de transformaciones profundas.

En resumen, a través de una breve revisión de los datos de opinión pública disponibles, es posible plantear que el electorado chileno ha sufrido dos graduales cambios desde la transición a la democracia en 1989 hacia adelante. En primer lugar, el país se ha ido moviendo desde una sociedad muy conservadora a una cada vez más liberal en temas socioculturales. Esta transformación es similar a la vivida en Europa occidental y se explica tanto por la tesis de la 'revolución silenciosa' de Ronald Inglehart, relacionada con la continua modernización económica del país, como por la irrupción de nuevos actores que han tenido la capacidad de politizar la transformación valórica de la sociedad chilena. En segundo lugar, con el pasar de los años ha ido disminuyendo la tolerancia hacia los niveles de desigualdad existentes y, por lo mismo, han venido ganando terreno las demandas por una modificación del modelo económico hacia una lógica más socialdemócrata. Si bien es cierto que esta transformación es multicausal (basta pensar en factores como los escándalos que han afectado la legitimidad de la elite empresarial, la disminución del ritmo del crecimiento económico y la existencia de una sociedad más educada), un agente crucial ha sido la formación de nuevos actores sociales capaces de politizar la desigualdad y promover la implementación de políticas públicas redistributivas que necesariamente chocan con el modelo de desarrollo existente en el país (Castiglioni y Rovira Kaltwasser 2016). ¿Cuál ha sido la estrategia electoral que 
la derecha ha desarrollado para abordar estas dos transformaciones del electorado chileno? Esta es la pregunta que busca responder el siguiente apartado.

\section{LA EVOLUCIÓN DE LA OFERTA PROGRAMÁTICA DE LA DERECHA CHILENA}

En cierto sentido, es posible plantear que los partidos políticos funcionan como empresas que compiten por un mercado que está constituido por los votantes. Visto así, los partidos políticos elaboran una oferta que busca satisfacer las demandas de los ciudadanos y, en consecuencia, el ganador de las elecciones es aquella fuerza política que tiene la habilidad de comprender de mejor forma cuáles son las preferencias de los votantes. No es casualidad, entonces, que la ciencia política haya venido estudiando de manera comparada cómo evolucionan las propuestas programáticas de los partidos políticos, ya que estas últimas pueden ser concebidas como las ofertas que son brindadas para conectar con las demandas de la ciudadanía (Budge, Robertson y Hearl 1987; Budge 1994).

El proyecto más sistemático al respecto consiste en el Manifesto Project Database, ${ }^{1}$ el cual recolecta los programas de gobierno que elaboran los partidos políticos para las campañas y que luego son analizados por personas que previamente han aprendido un manual de codificación en torno a diversos temas que son propios del debate político (economía, relaciones internacionales, etc.). En otras palabras, el análisis permite determinar cuánto espacio le dedican los partidos políticos a distintos tópicos. A partir de dichos datos es posible construir una serie de indicadores para examinar las propuestas programáticas de los partidos políticos en perspectiva comparada. Inicialmente, la recolección y análisis solo abarcaba los países de Europa occidental, pero con el tiempo se ha ido ampliando la cobertura para incluir a Estados Unidos, Europa del este y, más recientemente, a América Latina.

Hay una extensa literatura que explica los detalles metodológicos del Manifesto Project Database (Budge et al. 2001; Klingemann et al. 2006; Volkens et al. 2013), y anteriormente ya he revisado las propues-

\footnotetext{
${ }^{1}$ Información detallada sobre este proyecto se puede encontrar en Manifesto Project Database (ver Bibliografía).
} 
tas programáticas de las dos coaliciones dominantes en Chile y de la derecha en particular (Madariaga y Rovira Kaltwasser 2019; Rovira Kaltwasser 2019). Por ello, lo que me interesa en este artículo es presentar una actualización con los datos de la última elección presidencial del año 2017, a través de los cuales se comprende de mejor manera el error de diagnóstico de la derecha chilena y la disyuntiva en la cual se encuentra hoy en día.

La principal evidencia empírica que me interesa ofrecer es el llamado 'índice RILE', el cual se construye a partir de una serie de distintos indicadores y luego se estandariza en valores que van desde +100 para la postura más a la derecha hasta -100 para la postura más a la izquierda (Laver y Budge 1992). Una ventaja de este índice es que trabaja con datos para una gran cantidad de países a lo largo del tiempo y esto permite establecer comparaciones. Teniendo en cuenta que los orígenes de la coalición de derecha que se establece en el país desde 1989 en adelante está compuesta por dos partidos políticos - Renovación Nacional (RN) y Unión Demócrata Independiente (UDI) - , que se caracterizan por la defensa de las reformas económicas de libre mercado instauradas por la dictadura de Pinochet, resulta interesante establecer una comparación con las otras dos fuerzas a nivel mundial que han pujado con la misma fuerza por reformas de libre mercado, a saber, el Partido Republicano en Estados Unidos y el Partido Conservador en Reino Unido. En consecuencia, el siguiente gráfico (7) muestra el índice RILE para esos tres casos. ${ }^{2}$

Como se puede observar en el Gráfico 7, en las primeras dos elecciones presidenciales desde el retorno de la democracia en Chile (1989 y 1993), la coalición entre RN y UDI elabora programas de gobierno con una posición de derecha muy similar a la del Partido Republicano

\footnotetext{
${ }^{2}$ Mientras que en Estados Unidos y Reino Unido los programas de gobierno son elaborados por partidos políticos, en el caso de Chile los programas de gobierno generalmente son elaborados por coaliciones políticas que incluyen a más de un partido. Debido a ello, los datos que acá se presentan se construyen a partir de los programas de gobierno para la primera vuelta de las elecciones presidenciales de la coalición de derecha entre RN y UDI (más Evópoli en el caso de la última elección). La única excepción es la elección del año 2005, cuando RN y UDI llevaron a sus propios candidatos presidenciales a la primera vuelta electoral (Sebastián Piñera y Joaquín Lavín, respectivamente). Por lo tanto, en el Gráfico 7 el índice de RILE para el año 2005 es el promedio entre el candidato de RN $(-1,78)$ y el de UDI $(+13,51)$.
} 
en Estados Unidos y del Partido Conservador en Reino Unido, sobre todo en lo respectivo a la promoción de la expansión del libre mercado y de la reducción del Estado de Bienestar. Cabe destacar que en estas dos elecciones la derecha chilena obtiene resultados catastróficos: mientras Hernán Büchi conquistó un 29,4\% de votación el año 1989, Arturo Alessandri consiguió un $24,4 \%$ de los votos el año 1993. Recién en el año 1999 la coalición entre RN y UDI logra forzar la realización de un balotaje y, como se muestra en el Gráfico 7, en dicha elección el programa de gobierno de la derecha experimenta una notable moderación. Esto no es una casualidad, sino que obedece a un proceso de aprendizaje al interior de la derecha que se gatilla por los magros resultados electorales anteriores (Madariaga y Rovira Kaltwasser 2019). En efecto, la campaña presidencial del año 1999 es un punto de inflexión en la derecha chilena, ya que el candidato de aquel entonces (Joaquín Lavín) establece un giro importante en los temas que se abordan (por ejemplo, necesidad de expandir el Estado de Bienestar) y esto logra generar una mejor sintonía con las demandas de la ciudadanía, lo cual a su vez se traduce en un mucho mejor resultado electoral. Como se ha planteado con anterioridad, el electorado chileno se ha ido volviendo más liberal culturalmente y menos tolerante con la desigualdad socioeconómica, de modo que es esperable que la derecha moderara sus propuestas programáticas para así poder aumentar su nivel de votación.

Aun cuando este proceso de moderación programática de la derecha chilena, que se mantuvo hasta el primer gobierno de Piñera (Fairfield y Garay 2017; Niedzwiecki y Pribble 2017), no dejó a todos sus líderes y votantes satisfechos, la obtención de mejores resultados electorales funcionó como una fórmula para apaciguar las disputas internas. Sin embargo, este escenario comienza a cambiar durante el segundo mandato de Michelle Bachelet. Recordemos que el año 2013 la candidata de la coalición de la derecha (Evelyn Matthei) obtuvo un magro $25 \%$ de los votos en la primera vuelta electoral y, por tanto, no pocos líderes de derecha empezaron a plantear que era necesario frenar la moderación y (re)establecer una agenda más de derecha para poder conquistar al electorado. Por su parte, el segundo gobierno de Michelle Bachelet contó con el apoyo del Partido Comunista y por primera vez la izquierda intentó realizar reformas más estructurales, tales como la legalización del aborto ante ciertas causales, la transformación de la 
política tributaria, la implementación de un sistema universitario financiado por el Estado y la modificación de la Constitución del país. Ante este nuevo escenario, un sector relevante de la derecha consideró que era necesario salir a defender sus propias ideas con fuerza y alejarse de cualquier intento de moderación programática. Quien mejor representa esta presión por radicalización es la Fundación para el Progreso, la que, junto a su principal referente intelectual (Áxel Kaiser), en tanto, se dedicaron a atacar al segundo mandato de Bachelet con tal histrionismo que llegaron a plantear que ella buscaba instaurar el modelo 'CastroChavista' en Chile.

Gráfico 7. POSICIÓN EN EL EJE DERECHA-IZQUIERDA (RILE) DE LA COALICIÓN DE CENTRODERECHA EN CHILE, EL PARTIDO REPUBLICANO EN EEUU Y EL PARTIDO CONSERVADOR EN REINO UNIDO

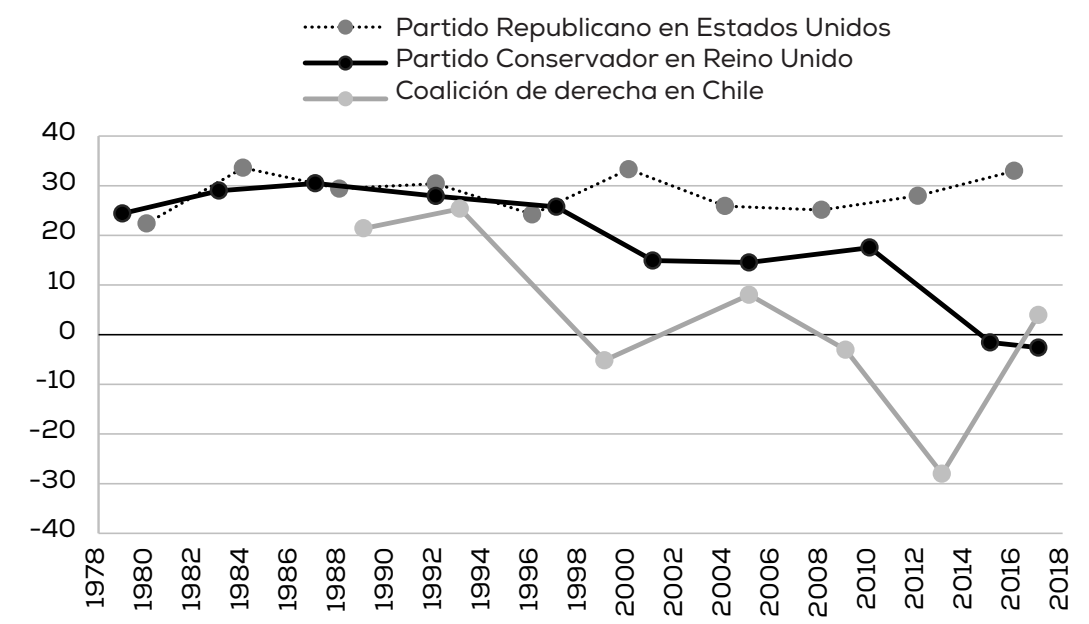

Fuente: elaboración propia en base a los datos del Manifesto Project Database.

Esta interpretación ganó mucho terreno al interior de los círculos de la derecha; a tal punto que, para la campaña presidencial del año 2017, el programa de gobierno de Sebastián Piñera gira marcadamente hacia la derecha y pone fin a la trayectoria de moderación programática. Como se puede observar en el Gráfico 7, el programa de gobierno de Piñera elaborado para la campaña del año 2017 está más a la derecha que los programas de gobierno elaborados por el Partido Conservador de Reino Unido en los años 2015 (David Cameron) y 2017 (Theresa 
May). Un análisis más detallado del programa de gobierno que Piñera elabora para la primera vuelta electoral del año 2017, permite comprender de mejor forma en qué áreas se abandona la moderación programática. Es por esto que en el siguiente gráfico (8) se muestra la relevancia que, desde 1989 en adelante, los candidatos presidenciales de derecha en Chile le dan en sus programas de gobierno a cuatro temas relacionados con disputas sobre el modelo económico, a saber: a) la expansión del libre mercado, b) la mayor regulación del libre mercado, c) la expansión del Estado de Bienestar y d) la reducción del Estado de Bienestar. ${ }^{3} \mathrm{Al}$ observar este gráfico, no solo queda en evidencia que el programa de Joaquín Lavín del año 1999 marca un punto de inflexión (caen de manera notoria las menciones a la expansión del libre mercado y aumentan de manera significativa las menciones a la expansión del Estado de Bienestar), sino que también se demuestra que el programa de gobierno de Sebastián Piñera del año 2017 no le da ninguna importancia a una mayor regulación del mercado (pese a los escándalos de colusión y del financiamiento de la política) y a su vez reduce el énfasis en la expansión del Estado de Bienestar.

Gráfico 8. RELEVANCIA DE TEMAS SOCIOECONÓMICOS EN LOS MANIFIESTOS DE LA COALICIÓN DE CENTRODERECHA EN CHILE (PORCENTAJE)

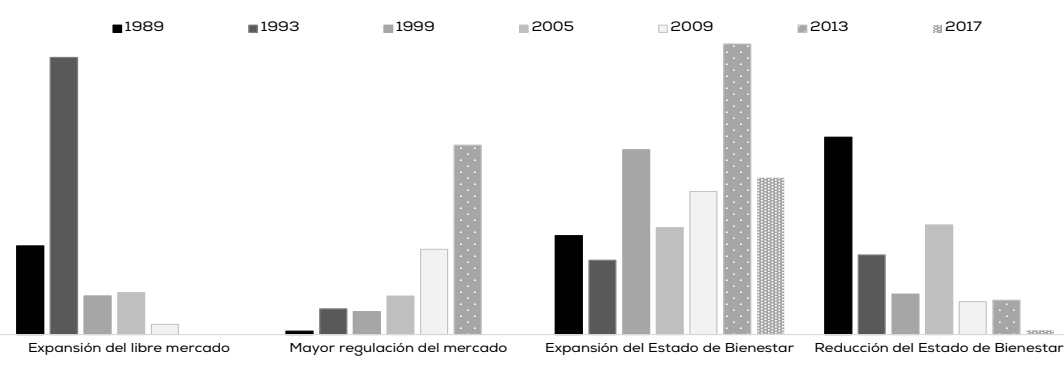

Fuente: elaboración propia en base a los datos del Manifesto Project Database.

Para comprender este giro de Piñera en su programa de gobierno del año 2017, es preciso recordar que para un sector importante de la

\footnotetext{
${ }^{3}$ Los valores para el año 2005 reflejan el promedio entre el candidato de RN (Sebastián Piñera) y el de UDI (Joaquín Lavín), ya que estos compitieron entre sí para la primera vuelta electoral.
} 
derecha, el segundo mandato de Michelle Bachelet fue visto como un período marcado por ideas extremistas y desapegadas de los intereses de la mayoría. No obstante, también es necesario tomar en cuenta un factor adicional: la irrupción de José Antonio Kast como candidato presidencial. Para las elecciones primarias de mediados del año 2017, la derecha compitió con tres candidatos (Felipe Kast, Manuel José Ossandón y Sebastián Piñera) y, más allá de ciertas tensiones entre ellos, el proceso sirvió para que RN, UDI y Evópoli respetaran el resultado final que dio como vencedor a Sebastián Piñera. Sin embargo, el diputado UDI José Antonio Kast decidió abandonar su partido y tomó la decisión de competir como candidato independiente, con el argumento de que era necesario atacar con dureza a la izquierda y adoptar posturas más radicales de derecha. No en vano, en su programa de gobierno, José Antonio Kast $(2017,1)$ indica que "[e]l gran error de la derecha en los últimos años ha sido renunciar a sus principios y acomodar sus valores para ganarse la simpatía de determinados sectores".

En efecto, la agenda que promueve José Antonio Kast tiene un tono apocalíptico muy similar al que desarrolla la Fundación para el Progreso y busca poner fin a la moderación programática de la derecha chilena, mostrando así una clara similitud con propuestas de la derecha populista radical de Europa occidental y la plataforma electoral de Donald Trump en Estados Unidos. ${ }^{4}$ Así, por ejemplo, en su programa de gobierno, Kast indica que es necesario "[r]evertir de manera inmediata, la Ley de Aborto que promueve Michelle Bachelet, enviando un proyecto para lograr su derogación" (Kast 2017, 14), como también menciona que se deben "[i]niciar estudios y comenzar la construcción de barreras físicas donde se requiera en la frontera Chile-Perú (170 kilómetros) y Chile-Bolivia (1.000 kilómetros)" (Kast 2017, 4), y en otra parte señala que es preciso "[i]nstaurar de manera permanente la convocatoria a un Comité de Seguridad Nacional que reemplace el Comité Político semanal en La Moneda y se aborde directamente la lucha contra el Terrorismo, el Narcotráfico y la Delincuencia" (Kast 2017, 8).

No está de más recordar que la agenda promovida por José Antonio Kast no solo recibió gran cobertura mediática, sino que también el beneplácito de importantes miembros de la elite del país. Un ejemplo

\footnotetext{
${ }^{4}$ Para un análisis detallado de la ideología de José Antonio Kast y su similitud con el perfil de la derecha populista radical, consultar Araya (2018).
} 
paradigmático al respecto fue su intervención en octubre del año 2017 en el foro empresarial de la Enade, en donde planteó un duro discurso que sacó ovaciones en el selecto público del Encuentro Nacional de la Empresa. De hecho, en un reportaje periodístico del evento se indica que "[d]e los 20 minutos que dura su exposición, José Antonio Kast no es interrumpido ni una, ni dos, sino 17 veces por los aplausos de los empresarios" (La Tercera 2017). Al levantar Kast una agenda de derecha populista radical que recibe bastante apoyo por parte de la elite económica, Sebastián Piñera, en conjunto con sus asesores, parece haber decidido elaborar una propuesta programática más enérgica de derecha, aun cuando los datos indicados anteriormente mostraban que el electorado chileno se había ido volviendo más liberal en temas culturales y más crítico de un modelo de libre mercado a ultranza. Este desarrollo es muy similar a lo que sucede en Europa occidental, en donde - como se indicó anteriormente- la emergencia y consolidación de partidos de derecha populista radical provocan una fuerte tensión al interior de la derecha convencional y esta última muchas veces opta por radicalizarse en vez de moderarse, con la falsa ilusión de que esto servirá para recuperar votantes desencantados.

¿Cuál fue el resultado de este giro programático adoptado por Piñera? Los datos de la primera vuelta electoral son bastante fehacientes al respecto. Aun cuando las encuestas daban una clara mayoría a Sebastián Piñera y hubo quienes incluso sugirieron que podía ganar en primera vuelta, la realidad fue bastante decepcionante para la derecha. Piñera obtuvo un $36,6 \%$ del electorado, lo cual equivale a aproximadamente 2,5 millones de votos. Cabe recordar que en la primera vuelta electoral del año 2009 Piñera logró un 44\% del electorado, lo cual equivale a aproximadamente 3,1 millones de votos. En otras palabras, si comparamos la primera vuelta de las elecciones de los años 2017 y 2009, Piñera no incrementó, sino que disminuyó su volumen electoral en más de medio millón de votantes.

Uno de los pocos dirigentes de la derecha que comprendió el error de moverse en exceso hacia la derecha, fue el senador Manuel José Ossandón, quien solo concedió su apoyo a Piñera para la segunda vuelta electoral, cuando este último estuvo dispuesto a transar ciertos puntos de su programa de gobierno, como por ejemplo al asegurar que no iba a tocar la reforma educacional de Bachelet que ofrece educación universitaria gratuita para los primeros seis deciles de la población, e incluso 
tuvo que comprometerse a ampliar esta política hacia la educación técnico-profesional. Por su parte, José Antonio Kast endosó sus votos a Piñera de manera rápida y sin condiciones, ya que a su juicio una victoria del candidato de izquierda (Alejandro Guillier) traería consigo la implantación del modelo 'Castro-Chavista' en Chile.

Como bien es sabido, Sebastián Piñera terminó ganando la segunda vuelta electoral efectuada el día 17 de diciembre del 2017 con una holgada mayoría de 54,6\%. Gran parte de la derecha consideró este resultado como un claro mandato, aun cuando los datos de la primera vuelta electoral fueron bastante mediocres y no se obtuvo una mayoría en el Congreso. Peor aún, la derecha pasó completamente por alto una peculiaridad de la democracia chilena: la dramática caída de la participación electoral que ha experimentado el país en el último tiempo. A nivel comparado, Chile presenta una de las mayores bajas en la participación electoral en el mundo (PNUD 2017, 11) y la evidencia empírica demuestra que la reforma del año 2012, que marca el tránsito desde el voto obligatorio al voto voluntario, reforzó aún más el abstencionismo electoral. El siguiente gráfico, que presenta los niveles de participación en las elecciones desde 1989 en adelante, es bastante elocuente al respecto.

Gráfico 9. PARTICIPACIÓN EN ELECCIONES PRESIDENCIALES EN CHILE POSTRANSICIÓN (EN PORCENTAJE)

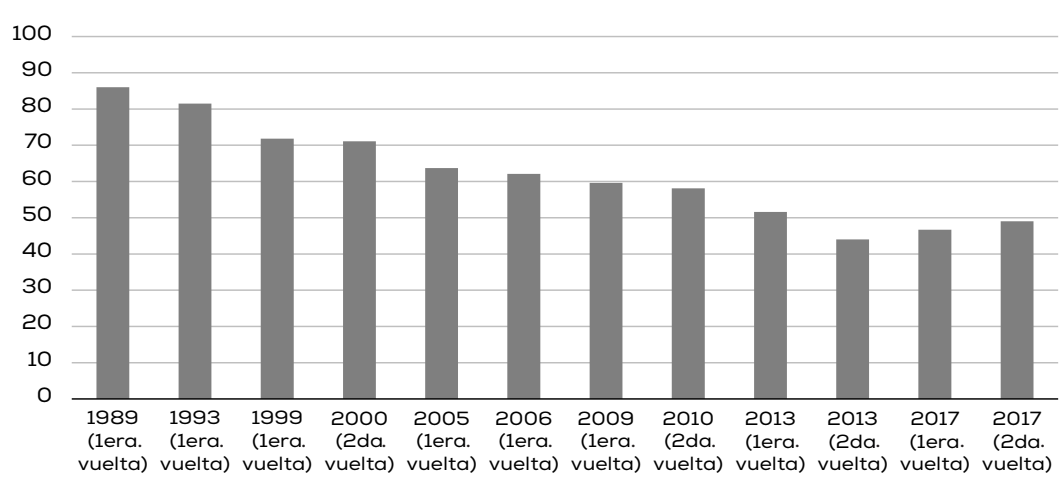

Fuente: elaboración propia en base a PNUD $(2020,106)$.

En resumen, Piñera y gran parte de la derecha cometió el mismo error que Bachelet y gran parte de la izquierda cuando esta última fue reelecta el año 2013: pensar que obtener una mayoría relativa, cuando 
participa aproximadamente la mitad del electorado, equivale a tener un contundente mandato de parte de la totalidad de la ciudadanía. Como veremos a continuación, este pensamiento, sumado al giro hacia la derecha del segundo gobierno de Piñera, debido tanto a la presión de una derecha populista radical como a la obsesión de culpar de todos los males del país al gobierno anterior, llevó a que la nueva administración adoptara una agenda gubernamental sumamente alejada de las preferencias de la ciudadanía, facilitando así el estallido social de fines del año 2019.

\section{EL ERROR DE DIAGNÓSTICO DE LA DERECHA CHILENA Y EL ESTALLIDO SOCIAL}

Piñera asume su segundo gobierno en marzo del año 2018 y su gran promesa de campaña consistió en señalar que 'se vienen tiempos mejores'. Dicho eslogan se basaba en la idea de que el nuevo gobierno iba a generar mejores condiciones económicas y que a su vez iba a enmendar el rumbo trazado por la administración anterior. Ahora bien, dado que el gobierno de Piñera no cuenta con mayoría en el Congreso, era de esperar que se potenciaran liderazgos más moderados y dialogantes. Sin embargo, la nominación de los ministros ya fue una primera señal que indicaba el error de diagnóstico del presidente y su círculo de hierro. Haciendo más caso a la presión por radicalización que por moderación e innovación, Piñera tomó la decisión de poner a varias figuras de su primer gobierno, como Andrés Chadwick a cargo del Ministerio del Interior y Felipe Larraín a cargo del Ministerio de Hacienda. A su vez, tres ministros del nuevo gobierno de Piñera provinieron de la Fundación para el Progreso: Roberto Ampuero a cargo del Ministerio de Relaciones Exteriores, Gerardo Varela a cargo del Ministerio de Educación y Mauricio Rojas a cargo del Ministerio de las Culturas, las Artes y el Patrimonio. El paso de estos tres ministros fue mediocre y sirvió para reforzar la falta de sintonía del gobierno con la opinión de la ciudadanía. Mientras Ampuero no ejerció liderazgo alguno a nivel internacional y fue monitoreado constantemente por el presidente, Varela se transformó en un verdadero dolor de cabeza para el gobierno por sus constantes polémicas como, por ejemplo, cuando señaló que "sus hijos eran unos campeones porque necesitaban muchos condones", calificó como "pe- 
queñas humillaciones" los casos de violencia de género puestos en el debate público por el movimiento feminista y argumentó que los colegios deberían "hacer bingos" para solucionar las goteras de las salas de clases (Cooperativa 2018a). Más penoso aún fue el paso de Rojas por el Ministerio de las Culturas, las Artes y el Patrimonio, ya que duró tan solo cuatro días en el cargo, puesto que se hicieron de conocimiento público sus dichos respecto de que el Museo de la Memoria no solo contaría una "versión falsa de la historia de Chile", sino que además sería un lugar diseñado "para que la gente no piense" y donde no se ofrece "ninguna explicación de cómo se llegó adonde llegamos" (Cooperativa 2018b).

Sumado a las polémicas de sus ministros, la situación económica no despegó como se había prometido. Al contar con una minoría en el Congreso y al seguir empecinado con la idea de tener un claro mandato para gobernar hacia la derecha, Piñera pudo avanzar muy poco en el Parlamento. El resto de la historia es bastante conocida a estas alturas. A inicios de octubre del año 2019 se toma la decisión de subir en treinta pesos el precio del boleto del Metro de Santiago y grupos organizados de estudiantes llamaron a no aceptar esta medida. Es así como rápidamente gran cantidad de personas comienzan a evadir el pago del Metro y el gobierno - apegado a su ceguera - opta no solo por inicialmente advertir que no se bajaría el valor anunciado, ${ }^{5}$ sino también por movilizar a Carabineros para reprimir a los manifestantes. Como si fuese poco, por esos días el ministro de Hacienda invitaba a la ciudadanía a comprar flores ante la baja de su precio, según los datos de la inflación, mientras que el ministro de Economía recomendaba a los ciudadanos de Santiago levantarse más temprano para aprovechar la tarifa baja del Metro. Esta falta de tino es un don tanto de varios de los ministros como del mismo presidente Piñera, quien cuando el día 18 de octubre comienza el estallido social por todo el país, encuentra natural salir a comer con su familia a una pizzería ubicada en una de las comunas más ricas del país.

Hoy en día no cabe duda alguna de que Chile cambió de manera profunda a contar del estallido social de fines de octubre del año 2019. Este fenómeno se explica en gran parte por el error de diagnóstico

\footnotetext{
${ }^{5}$ De hecho, unos días después del estallido social el gobierno terminó retrocediendo y tomó la decisión de congelar el alza del precio del boleto del Metro de Santiago.
} 
del segundo gobierno de Sebastián Piñera. Tan cierto es esto que las primeras acciones del presidente reflejan que tanto él como su círculo íntimo nunca entendieron adecuadamente lo que estaba sucediendo y por ello es que ante las masivas protestas toma la decisión de escribir un célebre discurso, en donde señala que "Chile está en guerra". En vez de intentar entender el malestar ciudadano y modificar su agenda, el presidente opta por seguir apegado a la hoja de ruta trazada en su programa de gobierno y es así como moviliza a las Fuerzas Armadas para intentar imponer orden. El desenlace de esta tozudez ha sido nefasto tanto para la administración de Piñera como para el país. Por un lado, los informes de organizaciones como el Alto Comisionado de las Naciones Unidas para los Derechos Humanos, Amnistía Internacional y Human Rights Watch muestran que los organismos de seguridad del Estado de Chile han cometido un número sumamente elevado de violaciones a los derechos humanos. Por otra parte, el gobierno se ha visto en la obligación de cambiar muy a regañadientes su agenda, lo cual se refleja sobre todo en el así llamado "Pacto por la paz social y una nueva Constitución", en donde se fija una hoja de ruta para cambiar la Constitución del país.

Si bien es cierto que existen pocos datos de opinión pública sobre el estallido social, la información existente refuerza la idea de que las preferencias de la ciudadanía son contrarias a las que ha venido defendiendo la administración de turno y que el gobierno de Piñera ha actuado de manera desastrosa. La última encuesta del Centro de Estudios Públicos (CEP 2019) es bastante iluminadora en este sentido, ya que según esta tan solo un $6 \%$ de la ciudadanía aprueba la gestión de Sebastián Piñera (el número más bajo en la historia desde el retorno de la democracia); más de la mitad de la población opina que las pensiones es el problema al que el gobierno debería dedicar mayor esfuerzo; dos tercios de la sociedad se muestran a favor del cambio constitucional, y un número similar opina que los carabineros violaron los derechos humanos durante la crisis que comenzó en octubre de 2019. Por su parte, la segunda encuesta Termómetro Social (2019), llevada a cabo a fines del año pasado, muestra no solo que tres cuartos de la ciudadanía están de acuerdo con el movimiento social, sino también que dos tercios de la sociedad están de acuerdo con que continúen las movilizaciones. 


\section{CONCLUSIONES}

Las transformaciones que ha venido experimentando la sociedad chilena representan un desafío mayúsculo para los partidos políticos, los cuales además enfrentan un complejo escenario producto del estallido social de fines del año 2019. Este artículo se ha focalizado en la situación de la derecha, la cual cometió un profundo error de diagnóstico respecto de cuáles son las demandas de la ciudadanía y de qué forma deben ser encausadas. A su vez, un argumento central de esta contribución es que la derecha chilena se encuentra hoy en día en una encrucijada similar a la que atraviesan los partidos de derecha convencional en Europa: por un lado, existe una presión por moderación programática para así intentar acercarse a la postura del electorado más de centro; por otro lado, se observa también una presión por radicalización programática para tratar de aproximarse al electorado más extremo. A modo de cierre, interesa tomar en consideración las ideas acá expuestas para reflexionar en torno al futuro de la derecha en Chile. Es por ello que a continuación se analizarán tres aspectos que resultan particularmente relevantes: la consolidación de un partido populista de derecha radical, la creciente tensión al interior de los partidos que forman parte del gobierno actual y, por último, la posibilidad de que el día de mañana se conformen nuevos tipos de coaliciones políticas.

En primer lugar, todo indica que José Antonio Kast y el Partido Acción Republicana llegaron para quedarse. Esto quiere decir que Chile cuenta hoy en día con un partido populista de derecha radical que elabora ideas muy similares a las que promulgan líderes como Jair Bolsonaro en Brasil y Donald Trump en Estados Unidos. Dada la radicalidad ideológica de este partido político, muy difícilmente podrá crecer de manera exponencial, sino que más bien está destinado a movilizar a un segmento particular del electorado. No obstante, producto del extremismo de este partido y de su líder, sus ideas marcadas por la combinación de autoritarismo, nativismo y populismo van a seguir acaparando la atención de la opinión pública y esto facilitará que tengan una influencia significativa en el sistema político chileno. De hecho, la literatura comparada sobre los partidos populistas de derecha radical indica que estos tienen una alta capacidad de modificar el debate público e incluso la agenda gubernamental, aun cuando su relevancia electoral 
sea reducida (Mudde y Rovira Kaltwasser 2017) e incluso generen un alto nivel de rechazo en gran parte de la ciudadanía (Rovira Kaltwasser, Vehrkamp y Wratil 2019).

En segundo lugar, las disputas entre los partidos políticos que forman parte del actual gobierno van a ir en aumento, no solo por la presión por radicalización que experimentan desde la derecha extrema liderada por José Antonio Kast y Acción Republicana, sino también por la aparición de dirigentes que demandan una moderación programática (Alenda 2020). En efecto, figuras como Mario Desbordes, Joaquín Lavín y Manuel José Ossandón han sido bastante francos en su posición contraria a la defensa del statu quo que postula gran parte de la derecha. Esta tensión entre moderación y radicalización se tornará particularmente complicada debido al proceso de cambio constitucional acordado después del estallido social. No es casualidad que UDI, RN y Evópoli hayan terminado dando libertad de acción a sus militantes respecto de cómo votar para el referéndum sobre el cambio constitucional, y lo más probable es que estas disputas se intensifiquen en caso de que el referéndum sea aprobado, ya que entonces comenzará un arduo debate respecto del modelo de desarrollo que conviene implementar. A su vez, todavía quedan dos años de gobierno, los cuales estarán marcados por discusiones y negociaciones en el Parlamento para hacer reformas estructurales en diversas áreas. Puesto que los partidos de derecha no tienen mayoría en el Congreso, su única opción es lograr moderar sus propuestas para conseguir votos de la oposición y esto sin duda alguna que dejará descontenta al ala más radical de la derecha.

En tercer y último lugar, para comprender el futuro de la derecha chilena es necesario observar también lo que sucede con el otro lado del espectro político. La izquierda se encuentra sumamente fragmentada y el estallido social ha reforzado esta tendencia centrífuga, de manera tal que es prácticamente imposible que todos los partidos que hoy en día están en la oposición puedan articular un bloque electoral capaz de sustentar un gobierno. Particularmente difícil es la situación de la Democracia Cristiana, un partido que, debido a la naturaleza del proceso de transición a la democracia chileno, terminó estableciendo una alianza electoral con partidos de centro-izquierda. Sin embargo, la literatura en política comparada categoriza a la familia de partidos de la Democracia Cristiana como partidos de centro-derecha (van Kersbergen 1995; 
Kalyvas y van Kersbergen 2010; Gidron y Ziblatt 2019) y, por tanto, no deberíamos extrañarnos de que la Democracia Cristiana chilena termine dividiéndose en dos grupos: uno de derecha y otro de izquierda. ${ }^{6}$ Visto así, lo más probable es que el país experimente en un futuro cercano un reordenamiento del sistema de partidos políticos, lo cual puede llevar a la conformación de nuevas coaliciones electorales. En efecto, el sector más de derecha de la Democracia Cristiana no debería tener grandes dificultades en apoyar explícitamente y/o implícitamente a un gobierno liderado por las fuerzas de derecha que apuestan por la moderación. Esto implicaría en todo caso un aislamiento de la tendencia más extrema liderada hoy en día por José Antonio Kast. Esto, salvo que, por supuesto, la derecha chilena esté preparada para sufrir un suicidio democrático al adherir a una opción radical como ha terminado aconteciendo en Estados Unidos bajo el liderazgo de Donald Trump.

\section{BIBLIOGRAFÍA}

Alenda, S. 2020. Introducción. Chile Vamos: anatomía de un proyecto de poder (1539). En Alenda, S. (ed.), Anatomía de la derecha chilena: Estado, mercado y valores en tiempos de cambio. Santiago: Fondo de Cultura Económica.

Araya, N. 2018. La emergencia de José Antonio Kast: ¿un caso de populismo de derecha radical en Chile? Tesis de Licenciatura en Ciencia Política, Universidad Diego Portales.

Bale, T. 2003. Cinderella and her Ugly Sisters: The Mainstream and Extreme Right in Europe's Bipolarising Party Systems. West European Politics 26(3), 67-90.

Budge, I. 1994. A New Spatial Theory of Party Competition: Uncertainty, Ideology and Policy Equilibria Viewed Comparatively and Temporally. British Journal of Political Science 24(4), 443-467.

Budge, I., Klingemann, H.-D., Volkens, A., Bara, J. y Tanenbaum, E. (eds.) 2001. Mapping Policy Preferences: Estimates for Parties, Electors, and Governments, 1945-1998. New York: Oxford University Press.

Budge, I., Robertson, D. y Hearl, D. (eds.) 1987. Ideology, Strategy and Party Change: Spatial Analyses of Post-War Election Programmes in 19 Democracies. New York: Cambridge University Press.

\footnotetext{
${ }^{6}$ En cierto sentido, desde hace un tiempo ya se puede observar este proceso de descomposición en distintas facciones como, por ejemplo, cuando Mariana Aylwin junto a varios connotados políticos de la Democracia Cristiana renunciaron al partido y crearon el movimiento Progresismo con Progreso. A su vez, es evidente que la gran mayoría de la actual bancada de los senadores de la Democracia Cristiana se siente mucho más cercana a la izquierda que a la derecha.
} 
Castiglioni, R. y Rovira Kaltwasser, C. 2016. Introduction. Challenges to Political Representation in Contemporary Chile. Journal of Politics in Latin America 8(3), 3-24.

CEP 2019. Encuesta Nacional de Opinión Pública No 84. Disponible en: https:// www.cepchile.cl/cep/encuestas-cep/encuestas-2009-2018/estudio-nacional-deopinion-publica-n-84-diciembre-2019 [23 de marzo 2020].

Cooperativa 2018a. No solo el bingo: las polémicas frases del ministro Varela. Disponible en: https://www.cooperativa.cl/noticias/pais/educacion/no-solo-elbingo-las-polemicas-frases-del-ministro-varela/2018-07-23/115046.html [23 de marzo 2020].

Cooperativa 2018b. Ministro de las Culturas, Mauricio Rojas, presentó su renuncia al gobierno. Disponible en: https://www.cooperativa.cl/noticias/pais/gobierno/ gabinete/ministro-de-las-culturas-mauricio-rojas-presento-su-renuncia-algobierno/2018-08-13/113143.html [23 de marzo 2020].

Donoso, S. y Von Bülow, M. (eds.) 2017. Social Movements in Chile. Organization, Trajectories, and Political Consequences. New York: Palgrave Macmillan.

Fairfield, T. y Garay, C. 2017. Redistribution under the Right in Latin America: Electoral Competition and Organized Actors in Policymaking. Comparative Political Studies 50(14), 1871-1906.

Gidron, N. y Ziblatt, D. 2019. Center-Right Political Parties in Advanced Democracies. Annual Review of Political Science 22(1), 17-35.

Ignazi, P. 1992. The Silent Counter-Revolution. Hypotheses on the Emergence of Extreme Right-Wing Parties in Europe. European Journal of Political Research 22(1), 3-34.

Inglehart, R. 1977. The Silent Revolution: Changing Values and Political Styles among Western Publics. Princeton: Princeton University Press.

Inglehart, R. 1990. Culture Shift in Advanced Industrial Society. Princeton: Princeton University Press.

Kalyvas, S. y van Kersbergen, K. 2010. Christian Democracy. Annual Review of Political Science 13(1), 183-209.

Kast, J.A. 2017. Un programa para volver a creer. Programa de gobierno. Disponible en: https://www.servel.cl/wp-content/uploads/2017/09/Programa_ Jose_Antonio_Kast_Rist.pdf [23 de marzo de 2020].

Kitschelt, H. 1994. The Transformation of European Social Democracy. New York: Cambridge University Press.

Klingemann, H.-D., Volkens, A., Bara, J., Budge, I. y McDonald, M.D. 2006. Mapping Policy Preferences II. Estimates for Parties, Electors, and Governments in Eastern Europe, European Union, and OECD (1990-2003). New York: Cambridge University Press.

Kriesi, H., Grande, E., Lachat, R., Dolezal, M., Bornschier, S. y Frey, T. 2008. West European Politics in the Age of Globalization. Cambridge: Cambridge University Press.

La Tercera 2017. El incómodo Kast. Disponible en: https://www.latercera.com/ noticia/el-incomodo-kast/ [23 de marzo 2020]. 
Laver, M. y Budge, I. (eds.) 1992. Party Policy and Government Coalitions. New York: St. Martin's Press.

Madariaga, A. y Rovira Kaltwasser, C. 2019. Right-Wing Moderation, LeftWing Inertia and Political Cartelisation in Post-Transition Chile. Journal of Latin American Studies 1-29. Disponible en: https://doi.org/10.1017/ S0022216X19000932 [23 de marzo 2020].

Manifesto Project Database. Disponible en: https://manifesto-project.wzb.eu/ [23 de marzo 2020].

Mansuy, D. 2016. Nos fuimos quedando en silencio. La agonía del Chile de la transición. Santiago: Instituto de Estudios de la Sociedad.

Mudde, C. 2007. Populist Radical Right Parties in Europe. New York: Cambridge University Press.

Mudde, C. 2013. Three Decades of Populist Radical Right Parties in Western Europe: So What? European Journal of Political Research 52(1), 1-19.

Mudde, C. y Rovira Kaltwasser, C. 2013. Exclusionary vs. Inclusionary Populism: Comparing Contemporary Europe and Latin America. Government \& Opposition 48(2), 147-174.

Mudde, C. y Rovira Kaltwasser, C. 2017. Populism. A Very Short Introduction. Oxford: Oxford University Press.

Niedzwiecki, S. y Pribble, J. 2017. Social Policies and Center-Right Governments in Argentina and Chile. Latin American Politics and Society 59(3), 72-97.

PNUD 2017. Desiguales. Orígenes, cambios y desafíos de la brecha social en Chile. Santiago: Programa de las Naciones Unidas para el Desarrollo.

PNUD 2020. Diez años de la auditoría a la democracia. Antes del estallido. Santiago: Programa de las Naciones Unidas para el Desarrollo.

Roberts, K. 2016. (Re)Politicizing Inequalities: Movements, Parties, and Social Citizenship in Chile. Journal of Politics in Latin America 8(3), 125-154.

Rovira Kaltwasser, C. 2017. A Bitter Victory for the Chilean Right. Foreign Affairs, 13 de diciembre. Disponible en: https://www.foreignaffairs.com/articles/ south-america/2017-12-13/bitter-victory-chilean-right [23 de marzo de 2020].

Rovira Kaltwasser, C. 2019. La (sobre)adaptación programática de la derecha chilena y la irrupción de la derecha populista radical. Colombia Internacional 99, 29-61.

Rovira Kaltwasser, C., Vehrkamp, R. y Wratil, C. 2019. Europe's Choice: Populist Attitudes and Voting Intentions in the 2019 European Election. Gütersloh: Bertlesmann Foundation.

Termómetro Social 2. 2019. Encuestas. Microdatos. Disponible en: https://www. microdatos.cl/termometro-social-2 [23 de marzo 2020].

van Kersbergen, K. 1995. Social Capitalism: A Study of Christian Democracy and of the Welfare State. London: Routledge.

Volkens, A., Bara, J., Budge, I. y D. McDonald, M.D. 2013. Mapping Policy Preferences from Texts. Statistical Solutions for Manifesto Analysts. New York: Oxford University Press. EP 